\title{
CALR Exon 9 Deletion Mutation
}

National Cancer Institute

\section{Source}

National Cancer Institute. CALR Exon 9 Deletion Mutation. NCI Thesaurus. Code C129832.

A molecular genetic abnormality indicating the presence of a deletion mutation in exon 9 of the CALR gene. These deletion mutations are associated with primary myelofibrosis and may be predicative of a favorable survival prognosis. 